\title{
Cultural Inertia and Uniformity in Organizations
}

\author{
Juan D. Carrillo* \\ University of Southern California and CEPR \\ Denis Gromb \\ London Business School and CEPR
}

We analyze aspects of the structure of organizational culture. We show that old and culturally uniform organizations are prone to cultural inertia, that is, they are reluctant to adopt a different culture in response to a change in the environment. Cultural uniformity can be beneficial because the associated inertia ex post protects and therefore ex ante encourages culture-specific investments by agents. We also explore the model's implications for such issues as cultural uniformity within an organization, cultural heterogeneity across organizations, the destabilizing effect of growth and mergers, and the conflicts arising in the management of culture.

\section{Introduction}

Organizations develop specific cultures that can affect their performance. Business successes and failures have often been attributed to corporate cultures. Top management increasingly recognize the importance of corporate culture and adopt active approaches to defining cultural identity, protecting it, and orchestrating cultural change. The notion extends beyond the corporate realm to administrations, nonprofit organizations, etc. Despite its relevance, organizational culture remains rather ill-defined in economic theory. In fact, its resistance to a precise description is one of its rare uncontroversial attributes.

This article develops an economic model of organizational culture to address some questions that we view as key to understand this concept (see Crémer 1995). Why do similar organizations develop different cultures with different long-run economic performances? Why don't organizations with inappropriate cultures always adopt new cultures better suited to their environment? Why are organizations reluctant to let multiple cultures coexist? And when they do, why does this coexistence tend to generate conflicts?

Our model's main premises are the following. First, agents in an organization are more productive if they fit in the culture in which they operate. Therefore, organizations strive to select agents who are "good fits". This idea

\footnotetext{
*University of Southern California and CEPR. Email: juandc@usc.edu.

We thank the editor, Keith Crocker, and two anonymous referees for their helpful comments.
}

The Journal of Law, Economics, \& Organization doi:10.1093/jleo/ewm021

(C) The Author 2006. Published by Oxford University Press on behalf of Yale University. All rights reserved. For permissions, please email: journals.permissions@oxfordjournals.org 
captures Schein's (1986a) belief that “every organization is concerned about the degree to which people at all levels 'fit' into its structure." Second, the selection of agents is imperfect and takes place over time. Therefore, organizations accumulate capital over time in the form of a stock of agents who are good fits for the culture (or cultures) in which they operate. In that respect, we adopt the view of Prescott and Visscher (1980) of the organization as a storehouse of information or of organization capital, whose objective is to select and allocate agents efficiently over time. Third, to fit in, agents have to make investments that are to some extent culture specific. For example, they have to form new habits, acquire certain social skills, etc. all of which have costs. Note that this notion of organization capital is related to the concept of social capital developed by Bourdieu (1983) and Coleman (1988), and which has become widely studied in the sociology literature. In words of the political scientist Robert Putnam: "Whereas physical capital refers to physical objects and human capital refers to the properties of individuals, social capital refers to connections among individuals - social networks and the norms of reciprocity and trustworthiness that arise from them" (Putnam 2000, 19; see also Cohen and Prusak 2001, 4). A major difference, however, is that social capital emphasizes the direct externalities of cooperation, whereas organization capital is concerned with matching between the skills of the individuals and the environment in which they operate.

We begin by analyzing some of the determinants of cultural inertia. There is strong evidence that some organizations have cultures that are maladapted to their environment, and that this is reflected in their performance. ${ }^{1}$ The question arises as to why some organizations do not adapt to changed environmental conditions, that is, why some form of cultural inertia prevails.

Cultural inertia can arise naturally in our framework. Consider an organization whose agents undertake a number of tasks. Tasks are best performed by following a coherent set of procedures, informal rules of behavior, etc. which we generically call a culture. For concreteness, assume that all agents in the organization operate in a culture that builds on adaptability or flexibility, and call it culture $A$. Some agents are better suited for this culture than others. For instance, independent, unruly individuals who are more comfortable and productive if they can freely organize their own schedule are good fits for culture $A$. Over time, the organization screens agents, keeping these good fits, and thereby accumulating cultural-specific organization capital.

Suppose now that following a change in environmental conditions, $A$ is no longer the most appropriate culture under which to perform a fraction of tasks. Instead, these tasks would be best performed under a more bureaucratic or rigid culture, that we will call culture $B$. If the organization does not adapt, these tasks continue being performed under culture $A$, and the revenue they generate is reduced. If, instead, the organization decides to adapt, the tasks are performed under culture $B$. This means that some of the agents whom

1. See Kotter and Heskett (1992) for a detailed analysis of the interaction between economic performance and corporate culture in different organizations. 
the organization selected for their fit with culture $A$ must now operate under culture $B$. Alternatively, these agents can be fired and replaced with new ones who are better fits for culture $B$, for example, bureaucratic and dedicated nine-to-six employees. In either case, the information accumulated about the existing agents and their fit with culture $A$ is lost. The destruction of this organization capital constitutes a cost of adapting.

This remark has three interesting implications. First, the cost of adapting to a new (orthogonal) culture increases with how well the agents fit in the organization's current culture. If the level of organization capital is low, so is the opportunity cost of destroying some of that capital. Since the "average fit" between an organization's agents and the culture in which they operate improves over time, older organizations will be less prone to adapting to an environment change. That is, cultural inertia increases with age (Proposition 1). ${ }^{2}$ Second, the cost of adapting stems from the fact that the organization's agents who have been screened to fit in culture $A$ are not necessarily good fits for culture $B$. Therefore, the cost of adapting would be lower if the organization had already selected some agents on the basis of their being good fits for culture $B$. In fact, the cost of adapting to the change would be minimized if all the organization's agents were selected to fit in culture $B$. By symmetry, however, this would maximize the cost of adapting to environment changes in which some tasks are best performed under culture $A$. If, instead, the organization had some fraction of agents selected under both culture $A$ and $B$, it would be more prone to adapt to changes of both types. In other words, culturally homogeneous organizations are less likely to adapt than culturally diverse ones. ${ }^{3}$ That is, cultural inertia increases with cultural uniformity (Proposition 2). Third, since homogeneity locks organizations in their current culture, there is an interesting trade-off between flexibility and gambling. On the one hand, organizations may decide to remain flexible enough to adapt to any environment change. This is achieved by allocating agents to both cultures. On the other hand, organizations may bet on whether the new environment will build on one of the existing cultures and screen all agents to fit in that culture. Which strategy is optimal depends on the cost of restructuring relative to the value of organization capital (Proposition 3). Note that this allocation dilemma is due to an indirect externality effect: the more agents are allocated to one culture, the higher the organization capital lost if tasks are restructured. Understanding the determinants of cultural inertia can also shed some light on the observed cultural heterogeneity across otherwise similar organizations. Older organizations may be locked in a culture at the time of an environment

2. Hirshleifer and Welch (2002) study a related problem from an informational perspective. They show that excess inertia in organizations where individuals have bounded memory (they recall past actions but not past signals) is more likely to occur when the environment is stable and when the same action has been taken long enough.

3. Casual evidence suggests that a strong cost cutting, financially oriented strategy was a major cause of Northwest Airlines' inability to adapt to a new competitive environment after deregulation in 1979. In contrast, American Airlines' more flexible customer-oriented strategy helped its adaptation (see Kotter and Heskett 1992). 
change, whereas younger organizations will adapt. Organizations of similar age might also have begun with what were at the time different but equally attractive (uniform) cultures and have become locked in.

We then argue that an organization's cultural structure can mitigate or exacerbate incentive problems. We show that in organizations in which multiple cultures coexist, agents have weaker incentives to undertake culture-specific investments. Conversely, cultural uniformity is more conducive to such investments. Therefore, the gains associated with cultural diversity must be weighted not only against the costs of restructuring but also against the cost of reduced incentives.

In our setup, the costs and benefits of cultural uniformity are two sides of the same coin. On the one hand, cultural uniformity increases the expected cost of adapting to an environment change. Hence, cultural diversity can be beneficial in that it gives the organization a greater ability to adapt to new contexts. On the other hand, the cultural inertia associated with cultural uniformity ex post protects and thus ex ante encourages agents' culture-specific investments. Therefore, cultural uniformity can also be desirable (Propositions 4 and 5). ${ }^{4}$ This second consideration is important when incentive schemes based explicitly on investment are not possible or excessively costly, and when the organization has no other way to commit not to adapt to an environmental change (e.g., through an explicit contract).

This point has several applications. First, an active management of organizational culture is key to controlling the tensions between the organization's performance and the welfare and incentives of its agents. These very tensions can generate conflicts. For instance, the organization and its agents may disagree about the optimal degree to which new agents should be screened. Indeed, more intense screening accelerates the process of inertia as the "average fit" between the agents and the culture in which they operate increases faster (i.e., organization capital is accumulated faster), implying a higher opportunity cost of adapting. Hence, if sorting is costly, it is possible that agents will favor more intense screening than the organization would be willing to pay for. Similar conflicts can also arise over the organization's hiring policy between agents of different age within the organization and also between agents working under different cultures.

A second application regards the effect of growth. The gains from cultural uniformity and accumulation of organization capital are endangered in fast growing organizations. The substantial influx of new hires dilutes the agents' cultural fit and so reduces the organization's opportunity cost of adapting. We argue that this consideration can constrain the organization's growth. Similarly, the stability ensured by cultural uniformity may be put at risk in mergers of culturally different organizations.

4. Boyer and Robert (2006) analyze a related trade-off in a principal-agent context with explicit contracts. They characterize the optimal contract when the agent's ex ante incentives to put effort in a project are affected by the principal's ex post flexibility to abandon the project if a more attractive one appears. 
Formal models of corporate culture have stressed the benefits of a strong cultural identity. For instance, it can accelerate the organization's reputation building (Kreps 1990) or encourage horizontal communication (Crémer 1993). Other recent works have focused on the importance of cultural uniformity. We depart from these mainly in two respects. First, in the informal literature (Kotter and Heskett 1992), rigid cultures are always suboptimal in uncertain environments but can nonetheless prevail due to the inability of managers to impose more flexible ones. Also, some cultures may prove inferior to others and yet be maintained because superior cultures are inimitable (Barney 1986; Besanko et al. 2000). Instead of assuming the impossibility of a cultural change, cultural inertia arises in our article as the endogenously optimal response to incentive problems within the organization. Second, most formal theories (Lazear 1999; Rob and Zemsky 2002) posit a direct positive externality between agents with similar cultures. ${ }^{5}$ In contrast, we assume away such direct externalities to better point out an indirect one: the more agents fit in a given culture, the higher the opportunity cost for the organization to undertake changes such that this organization capital is lost. ${ }^{6}$ Finally, our article is related to the literature on incentives and corporate structure. In this context, Rotemberg and Saloner (1994) make the related point that a narrow business strategy enables firms to motivate employees to search for ways of increasing productivity in its core activities.

The article proceeds as follows. Section 2 presents a model of organizational culture and derives some of its basic properties, including the effect of time on the organization's incentives to adapt to changed environmental conditions. Section 3 studies how the cultural structure of an organization (heterogeneous or homogeneous) also affects cultural inertia. Section 4 shows how cultural uniformity can mitigate the agents' incentive problems. Section 5 develops implications of the theory for conflicts over the management of cultures and for the impact of growth and mergers on the cultural stability of an organization. It also suggests possible extensions. Section 6 concludes. Our central conclusions are gathered in Propositions 1-5. Some other interesting insights can be found in Results 1-7. All proofs are relegated to the Appendix.

\section{A Model of Organizational Culture}

In this section, we develop a model of organizations and organizational culture (Section 2.1). We begin by introducing two of the three main features of our

5. For instance, agents might interact better or reciprocate more if they share the same culture. However, it is unlikely that all agents in large organizations interact with each other directly or even through third parties. Why should loosely connected parts of an organization have similar cultures?

6. We do not mean that direct interactions are irrelevant: the literature on social capital widely documents the existence of complementarities between individuals. However, this restriction allows us to stress the idea that, even in a context where cultural diversity is a superior organizational structure, the agents' incentive problem may induce the organization to adopt a uniform culture. 
model. First, an organization's agents are more productive if they fit in the culture in which they operate. Second, organizations select agents who are good fits but do so imperfectly and over time. ${ }^{7}$ This setup captures the idea that organization capital accumulates over time (Section 2.2), and therefore that time is a determinant of cultural inertia (Section 2.3).

\subsection{The Basic Model}

We consider an organization with the following characteristics.

Tasks. The organization performs a continuum of tasks (with measure 1) in each of an infinite number of periods $t=0,1,2, \ldots$ Each task requires exactly one agent.

Payoffs. Agents receive a fixed wage $w$ in each period of employment by the organization. The outside wage is normalized to 0 . The total payoff for the organization and the agents is the sum of all their per-period payoffs discounted at the same rate $\delta$.

Cultures. A given task is best performed by following a coherent set of procedures, informal rules of behavior, etc. which we generically call a "culture". We assume that there are only two possible cultures in the economy, $A$ and $B$. For concreteness, one can think of culture $A$ as building on "adaptability", whereas culture $B$ is more "bureaucratic".

Cultural fit. Some agents are good fits for a culture, whereas others are "misfits". For instance, good fits for a culture based on adaptability may be independent and unruly individuals productive if they can organize their schedule. Conversely, bureaucratic, dedicated nine to six types, productive when their mission is well specified will be better fit for a bureaucratic culture.

Labor market. There is a labor market in which a fraction $s$ of agents are good fits for culture $A$ and a fraction $s$ of agents are good fits for culture $B$. The overlap between these two sets, that is, the agents who are good fits for both cultures, represents a fraction $s \mu$ of all agents in the labor market, where $\mu \in$ $(0,1)$. The parameter $\mu$ then measures the "closeness" of cultures $A$ and $B$. Last, the remaining (positive) fraction of agents are misfits for both cultures. ${ }^{8}$ For the time being, we assume that $s$ is fixed: agents cannot affect their likelihood of being good fits. This parameter will be endogenized in Section 4, where we consider culture-specific investments.

Revenues. Agents working in the organization are more productive if they fit in the culture in which they operate. Formally, in each period, a task

7. For now, we leave out the third main feature of our model, namely that to fit in, agents have to make costly investments that are to some extent culture specific. This feature is introduced in Section 4 .

8. Summing up, a fraction $s(1-\mu)$ are good fits only for culture $A$, a fraction $s(1-\mu)$ are good fits only for culture $B$, a fraction $s \mu$ are good fits for both $A$ and $B$ and a fraction $1-2 s+s \mu$ are misfits for both $A$ and $B$. 
generates revenue $R_{L}$ to the organization if performed by a misfit and $R_{H} \equiv R_{L}+\Delta$ if performed by a good fit. A task is best performed by a good fit (i.e., $\Delta>0$ ), but is profitable even if performed by a misfit (i.e., $R_{L}>w$ ).

Screening and turnover. The organization screens agents on the basis of whether they are good fits for the culture in which they operate. In period $t=0$, a continuum of agents (with measure 1) are hired from the labor market. At the end of each period, each agent quits the organization with probability $(1-\lambda)$. For simplicity, $\lambda$ is assumed to be exogenous and independent of the agent's identity and history. Among the remaining agents, each misfit is detected with probability $p$, and automatically fired. All departing agents (those who quit and those who are fired) are replaced with new agents from the labor market. Therefore, in each period, the organization employs a continuum of agents with measure $1 .{ }^{9}$

Contracts. Only short-term contracts are feasible. In particular, the organization cannot commit on severance payments or employment. Also, the number of periods an agent has spent in the organization is not observable. This allows us to avoid the issue of discriminatory firing.

\subsection{The Organization as Sieve}

For now and for simplicity, assume the existence of a unique culture, say culture $A$. In our model, the organization screens its agents so as to increase their fit with its culture. We denote by $s_{t}$ the organization's cultural fit in period $t$, defined as the fraction of its agents who are good fits for its culture. The population of agents in the organization evolves over time, due to screening and exogenous departures. Consider the organization at $t=0$. As all of its agents have just been hired from the labor market, the fraction of good fits is $s_{0}=s{ }^{10}$ At the end of period $t=0$, a fraction $(1-\lambda)$ of agents voluntarily quit the organization. Therefore, a mass $\lambda s$ of good fits and a mass $\lambda(1-s)$ of misfits remain. Finally, among the remaining mass $\lambda(1-s)$ of misfits, a fraction $p$ of them are screened out. Hence, the total mass of agents leaving the organization voluntarily or through firing is $(1-\lambda)+\lambda(1-s) p$. These agents are replaced by new hires, a fraction $s$ of whom are good fits. The organization's cultural fit in period $t=1$ is then

$$
s_{1}=\lambda s+[(1-\lambda)+\lambda(1-s) p] s>s_{0} .
$$

9. We rule out the possibility that the organization hires more agents than needed and screens unskilled agents so as to reduce the cost of replacing active ones. This option is never optimal in our model if screening occurs on the job, that is, if it is impossible or excessively costly to evaluate idle agents. Note also that, given no replacement cost, it is always optimal to fire a detected misfit. For models in which the organization first evaluates agents in screening tasks and then allocates them to productive jobs, see Prescott and Visscher (1980) and Carrillo (2003).

10. Of these agents, $s(1-\mu)$ are misfits for culture $B$ and $s \mu$ are good fits also for culture $B$ although, at this stage, their fit with culture $B$ is irrelevant. 
More generally, the relationship between the organization's cultural fit in periods $t$ and $t-1, s_{t}$ and $s_{t-1}$, is

$s_{t}=\lambda s_{t-1}+\left[(1-\lambda)+\lambda\left(1-s_{t-1}\right) p\right] s=\lambda(1-p s) s_{t-1}+(1-\lambda+\lambda p) s$.

Solving for the generic term of the series, we get

$s_{t}=\left[\frac{1-\lambda+\lambda p}{1-\lambda+\lambda p s}-\lambda^{t}(1-p s)^{t} \frac{\lambda p(1-s)}{1-\lambda+\lambda p s}\right] s$.

Note that the organization's cultural fit increases over time $\left(s_{t+1}>s_{t}\right)$. Besides, due to exogenous quits $(\lambda<1)$, there is always a fraction of misfits in the organization even in the long run $\left(s_{\infty}=\frac{1-\lambda+\lambda p}{1-\lambda+\lambda p s} s\right)<1$. Last, in the absence of screening $(p=0)$ or if every agent exogenously quit the organization after one period $(\lambda=0)$, the fraction of good fits would remain constant over time $\left(s_{t}=s\right.$ for all $\left.t\right)$.

In period $t$, the organization receives the fixed revenue $R_{L}$ from all of its agents and pays them a wage $w$. Also, good fits (in proportion $s_{t}$ ) generate an additional revenue $\Delta$. Hence, the organization's value from period $t$ onward (its continuation value at $t$ ) is

$V_{t}=\frac{R_{L}-w}{1-\delta}+\Delta \sum_{\tau=0}^{\infty} \delta^{\tau} s_{t+\tau}$

Note that screening acts as a sieve and increases the proportion of good fits over time. Therefore, the organization's continuation value also increases over time. For instance, given equations (1) and (2), the increase in continuation value between period 0 and period $t$ is

$V_{t}-V_{0}=\Delta \sum_{\tau=0}^{\infty} \delta^{\tau}\left(s_{t+\tau}-s_{\tau}\right)=\frac{\left(s_{t}-s_{0}\right) \Delta}{1-\delta \lambda(1-p s)}>0$,

which is also the deadweight loss incurred if the organization is dissolved in period $t$ and restarted from scratch. In other words, the organization accumulates capital over time in the form of a stock of agents who are good fits for the culture under which they operate. If the organization is terminated, its organization capital is lost. The result is summarized as follows.

Result 1 (Organization capital). As the organization ages, its cultural fit increases $\left(s_{t+1}>s_{t}\right.$ for all $\left.t\right)$ and so does its continuation value $\left(V_{t+1}>V_{t}\right.$ for all $t$ ).

\subsection{Time and Inertia}

As Wilson $(1989,91)$ nicely points: "Culture is to an organization what personality is to an individual. Like human culture generally, it is passed on from one generation to the next. It changes slowly, if at all." Numerous case studies document instances of cultural inertia, that is, cases in which an 
organization fails to adapt its culture to a new environment. ${ }^{11}$ Phenomena of cultural inertia arise naturally in our framework when we consider a changing environment.

Environment change. In a given period $T$, an environment change occurs, and the organization can decide whether to adapt. One can think of the environment change as the discovery of a new technology or a new production method. If the organization does not adapt (i.e., if it does not acquire this technology or production method), it loses its competitive edge: given this foregone opportunity, the revenue generated from period $T$ onward in a fraction $\gamma$ of the nonrestructured tasks decreases by an amount $\Delta^{\prime}$ (with $\Delta^{\prime}>\Delta$ ). That is, each of the $\gamma$ tasks generates $R_{L}-\Delta^{\prime}$ or $R_{H}-\Delta^{\prime}$ if performed by a misfit or a good fit for culture $A$, respectively. ${ }^{12}$ For simplicity, we assume that $T$ is known, that there is no further environment change afterward, and that all tasks are valuable even if the organization does not adapt $\left(R_{L}-\Delta^{\prime}>w\right)$.

Restructuring. Alternatively, the organization can adapt to the new environment and restructure the $\gamma$ tasks, which involves a fixed cost $K^{13}$ At the time of the decision on whether to adapt, the extent of the environment change (measured by $\gamma$ ) is not known. Instead, $\gamma$ is distributed over $[0,1]$ according to a known distribution $F(\cdot)$.

Cultural compatibility. If the organization decides to adapt, the $\gamma$ tasks are best performed by agents who are good fits for culture $C$. We distinguish between two cases: a culturally compatible restructuring, that is, one which builds on the existing culture (we denote it $C=A^{\prime}$ ), and a culturally incompatible restructuring, that is, one which builds on the opposite culture (we denote it $\left.C=B^{\prime}\right){ }^{14}$

Consider first the culturally compatible case $\left(C=A^{\prime}\right)$. It corresponds to a restructuring where independent, creative individuals (good fits for culture $A$ ) are suitable for the new tasks or production method. For example, this occurs if new missions are not well defined and the new tasks need the input and imagination of its agents. It is optimal for the organization to adopt this new production method if and only if the expected increase in the value of payoffs brought about by the restructuring exceeds its cost. Formally,

$\Gamma \equiv \frac{\Delta^{\prime}}{1-\delta} E[\gamma]-K>0$

In the remainder of the article, we assume that this condition holds, that is, a culturally compatible restructuring is always profitable.

11. For example, after several years of good performance, Xerox and Northwest Airlines failed to adapt their culture to a contextual change of increased competition due, respectively, to the emergence of new Japanese organizations and the deregulation of the airline industry. In contrast, young Japanese firms adapted successfully (see Kotter and Heskett 1992).

12. Identical results are obtained if revenues do not change under no restructuring and revenues increase by $\Delta^{\prime}$ in a fraction $\gamma$ of tasks under restructuring.

13. Given a fixed cost to adapt, it is optimal to restructure either none or all the $\gamma$ tasks.

14. It is not crucial for our analysis that culture $C$ builds entirely on either culture $A$ or culture $B$. It is only necessary that it be "closer" to one than to the other. 
The culturally incompatible restructuring case $\left(C=B^{\prime}\right)$ is slightly more complex. It corresponds to a new production method with well-defined tasks and rigid schedules, for which agents who are good fits for $B$ are suitable but agents who are good fits only for $A$ are not. Suppose that the organization decides to adapt to the change. Given $\Delta^{\prime}>\Delta$, it is more profitable to assign the $\gamma$ restructured tasks to culture $B^{\prime}$ immediately (i.e., at date $T$ ) rather than to keep agents working under culture $A$ and restructure the corresponding tasks only as these agents depart. However, the organization still has to decide whether to fire $\gamma$ agents and replace them with agents from the labor market (the "radical" strategy) or to set $\gamma$ existing agents currently screened for their fit to culture $A$ to work under culture $B^{\prime}$ (the "gradual" strategy).

Compared to the case $C=A^{\prime}$, the radical strategy when $C=B^{\prime}$ involves the additional cost of replacing an expected mass $E[\gamma]$ of agents screened under culture $A$ with unscreened agents, who will perform under culture $B^{\prime}$. Losing the screened agents has a cost proportional to $s_{T}$. However, not all the replacements will be misfits; a proportion $s$ of them will be good fits for the new culture $B^{\prime}$. Using equations (3) and (4), the net value of restructuring with the radical strategy is then

$\Gamma-\frac{\left(s_{T}-s\right) \Delta}{1-\delta \lambda(1-p s)} E[\gamma]$

The gradual strategy when $C=B^{\prime}$ also involves a cost compared to $C=A^{\prime}$. Reallocating the agents screened under $A$ has a cost proportional to $s_{T}$. However, since $\mu>0$, some of these agents will still be good fits for culture $B^{\prime}$. Formally, the fraction of good fits for culture $B^{\prime}$ at date $T$ and given that these agents have been screened for their fit to culture $A$ is ${ }^{15}$

$\tilde{s}_{T} \equiv s_{T} \mu+\left(1-s_{T}\right) \frac{s(1-\mu)}{1-s}$.

Therefore, using again equations (3) and (4), the net value of restructuring with the gradual strategy is

$\Gamma-\frac{\left(s_{T}-\tilde{s}_{T}\right) \Delta}{1-\delta \lambda(1-p s)} E[\gamma]$

or, using the above expression of $\tilde{s}_{T}$

$\Gamma-\frac{\left(\frac{1-\mu}{1-s}\right)\left(s_{T}-s\right) \Delta}{1-\delta \lambda(1-p s)} E[\gamma]$

By inspection of equations (5) and (6), we reach our second result.

15. This expression follows from the fact that $\operatorname{Pr}(B \mid A)=\frac{\operatorname{Pr}(B \cap A)}{\operatorname{Pr}(A)}=\frac{s \mu}{s}=\mu$ and $\operatorname{Pr}(B \mid \bar{A})=$ $\frac{\operatorname{Pr}(B \cap A)}{\operatorname{Pr}(A)}=\frac{s-s \mu}{1-s}$. 
Result 2 (Cultural incompatibility and inertia). A culturally incompatible restructuring is more likely to be adopted with a gradual rather than a radical strategy if cultures are close to each other (greater $\mu$ ) and there are few good fits in the labor market (smaller $s$ ).

The closeness of cultures and the availability of good fits in the labor market determine the relative value of the two restructuring strategies. More precisely, from equations (5) and (6) we notice that the gradual strategy outperforms the radical one if and only if $s<\mu$. Nevertheless, since both strategies involve the opportunity cost of a loss in organization capital, the best of them does too. The opportunity cost per task restructured under the best restructuring strategy is

$G_{T} \equiv \frac{1-\max \{s, \mu\}}{1-s} \frac{\left(s_{T}-s\right) \Delta}{1-\delta \lambda(1-p s)}$.

Therefore, the organization decides to adapt to the environment change if and only if

$\Gamma-G_{T} E[\gamma]>0$.

This expression captures the organization's trade-off when considering a culturally incompatible restructuring. On the one hand, the organization must consider the benefit of adapting to the new environment, which leads to higher revenues in the future. On the other hand, a culturally incompatible restructuring involves the cost of giving up part of the gains of past screening, that is, it implies the loss of some of the accumulated organization capital.

Proposition 1 (Determinants of inertia: time). Organizations that are older at the time of the environment change (greater $T$ ) and with more short-term interest (smaller $\delta$ ) are less prone to adopt a culturally incompatible restructuring.

\section{Proof. See Appendix A1.}

The population of agents in old organizations has been screened for a long period of time. As a result, they are on average highly suited to the existing culture. Therefore, the opportunity cost of losing the benefit of past screening increases over time. This leads to inertia increasing with age. ${ }^{16}$ Similarly, when the organization puts more weight on future revenues, the benefits of a culturally incompatible restructuring increase relative to its cost. Consequently, such a restructuring is more likely.

\section{The Structure of Organizational Culture}

In this section, we study the possible coexistence of multiple cultures within the same organization. We first examine the effects of an organization's

16. A similar inertia is analyzed in the literature on technological leapfrogging (see Brezis et al. 1993). 
cultural structure (i.e., the mix of cultures within the organization) on its adaptation to environment changes (Section 3.1), and then derive implications for the optimal choice of cultural structure (Section 3.2). To analyze these issues, we assume that the organization may decide to allocate different tasks to different cultures and to screen agents accordingly.

Multiple cultures. In period 0 , the organization allocates a fraction $\alpha$ of tasks to culture $A$ and a fraction $1-\alpha$ to culture $B$. It is immediately noticed that, since $\mu<1$, the organization will optimally keep constant the fraction of tasks under each culture. That is, each agent hired from the labor market is set to work under the same culture as the agent he replaces.

\subsection{Uniformity and Inertia}

Suppose that $C=B^{\prime}$. Building on Section 2.3, we know that restructuring has benefits and costs. First, an expected mass $E[\gamma]$ of tasks generate a per-period revenue increased by $\Delta^{\prime}$. Moreover, if $\gamma<1-\alpha$, the only cost to pay for this restructuring is $K$, since the agents allocated to the restructured tasks are already screened under the compatible culture $B$. However, if $\gamma>1-\alpha$, then a mass $\gamma-(1-\alpha)$ of tasks previously performed under culture $A$ will now be performed under culture $B^{\prime}$. As shown before, the culturally incompatible restructuring of these agents (whether gradual or radical) has an opportunity cost $G_{T}$. Overall, the value of restructuring is

$$
\Pi_{T}(\alpha) \equiv \Gamma-G_{T} \int_{1-\alpha}^{1}(\gamma-(1-\alpha)) \mathrm{d} F(\gamma) .
$$

Note that $\Pi_{T}(0)=\Gamma$ and $\Pi_{T}(1)=\Gamma-G_{T} E[\gamma]$. These correspond to expressions (4) and (8), respectively, that is, to a fully compatible and a fully incompatible restructuring. In other words, equation (9) generalizes the opportunity cost of a restructuring to organizations with an initial cultural mix $(\alpha \in(0,1))$. Note also that, given the symmetry of cultures $A$ and $B$, the value of restructuring when $C=A^{\prime}$ is simply $\Pi_{T}(1-\alpha)$. Cultural inertia is thus affected by the cultural structure of the organization.

Proposition 2 (Determinants of inertia: uniformity). Organizations are less prone to undertake a restructuring that is culturally incompatible for many of their tasks ( $B^{\prime}$ if $\alpha$ is high and $A^{\prime}$ if $\alpha$ is low).

\section{Proof. See Appendix A2.}

When more agents work under $A$, the benefit $\Gamma$ from restructuring is unchanged, but the opportunity cost if $C=B^{\prime}$ is higher $\left(\partial \Pi_{T}(\alpha) / \partial \alpha<0\right)$. Indeed, as fewer of the organization's agents already fit in culture $B$ when restructuring occurs with $C=B^{\prime}$, more tasks will have to be switched from culture $A$ to culture $B^{\prime}$, resulting in a larger loss in organization capital. 
The analysis so far has implications for the source of cultural diversity across organizations. First, older organizations may be more reluctant to adapt. That is, organizations of different age, but otherwise similar, may react differently to a change in their environment (Proposition 1). Another channel is that firms of similar age started off with and became locked in different but equally attractive cultures whose performance later diverged. Different organizations may operate under different cultural structures due, for instance, to different access to labor or different priors about the environment. The more culturally homogeneous ones may have become locked in (Proposition 2). In other words, cultural heterogeneity across organizations may be a consequence of cultural uniformity within organizations.

\subsection{Multiple Cultures: Flexibility versus Gambling}

So far, we have taken the organization's cultural structure as given. In practice, firms select different strategies with a different mix of cultures. For example, the policy of Coors in the $80 \mathrm{~s}$ was homogeneous and conservative; all agents were screened on the basis of their skills for improving quality, but never to follow an aggressive and risky research strategy. By contrast, Nissan developed a more flexible business policy: some employees were engaged in new, highly risky projects, whereas others continued the production of high quality but standard cars (see Kotter and Heskett 1992).

These choices are very much related to the anticipation of the organization's environment. To analyze this idea and its implications for cultural structure, we assume that until period $T$, the organization is uncertain about whether $C=$ $A^{\prime}$ or $C=B^{\prime}$. For simplicity, assume that both outcomes are equally likely, and let $\alpha^{*}$ denote the mass of agents working under culture $A$ that maximizes the organization's expected payoff. We can determine its optimal cultural mix.

\section{Proposition 3 (Flexibility versus gambling in the choice of culture).}

(a) If $\Pi_{T}\left(\frac{1}{2}\right)<\frac{1}{2} \Pi_{T}(0)$, a uniform culture is optimal $\left(\alpha^{*} \in\{0,1\}\right)$ and only a culturally compatible restructuring is undertaken (only when $C=A^{\prime}$ if $\alpha^{*}=1$ and only when $C=B^{\prime}$ if $\alpha^{*}=0$ ).

(b) Otherwise, it is optimal to maintain an equal number of tasks under both cultures $\left(\alpha^{*}=1 / 2\right)$ and adapt irrespective of the type of restructuring (both when $C=A^{\prime}$ and $C=B^{\prime}$ ).

\section{Proof. See Appendix A3.}

If the organization plans to restructure only if $C=A^{\prime}$, then it should put all its eggs in one basket and set $\alpha^{*}=1$. This is because the more the tasks are under culture $A$, the lower the expected opportunity cost of restructuring when $C=A^{\prime}$ (formally, $\left(\partial \Pi_{T}(1-\alpha) / \partial \alpha<0\right)$ ). Under such a strategy, the organization's expected payoff of a restructuring weighted by the likelihood of a restructuring is $\frac{1}{2} \Pi_{T}(0)$. 
Note, however, that the gains of having one more task under culture $A$ when $C=A^{\prime}$ is decreasing in the existing fraction of tasks under that culture (formally, $\left.\partial^{2} \Pi_{T}(1-\alpha) / \partial \alpha^{2}<0\right)$. Indeed, given that only a fraction $\gamma$ of tasks need to be restructured, if the mass of tasks under culture $A$ is sufficiently large, then it is already unlikely that the organization will need to switch any task from $B$ to $A^{\prime}$ after restructuring. As a result, the marginal value of having yet another task under $A$ decreases with $\alpha$. Due to these decreasing returns to scale, conditional on a restructuring occurring irrespective of whether $C=A^{\prime}$ or $C=$ $B^{\prime}$, it is optimal to set $\alpha^{*}=1 / 2$. The organization's expected payoff of a restructuring if it always adapts is then $\Pi_{T}(1 / 2)$.

Proposition 3 highlights a fundamental trade-off between "flexibility" and "gambling": organizations must decide either to remain adaptable enough to restructure both when $C=A^{\prime}$ and when $C=B^{\prime}$ or bet on whether $C=A^{\prime}$ or $C=B^{\prime}$ and allocate all tasks to the same culture. This allocation dilemma is due to an indirect externality: the more the agents are allocated to one culture, the higher the organization capital lost if tasks are restructured. Which strategy is optimal depends on the sign of

$$
\Pi_{T}\left(\frac{1}{2}\right)-\frac{1}{2} \Pi_{T}(0) \equiv \frac{1}{2} \Gamma-G_{T} \int_{1 / 2}^{1}(\gamma-1 / 2) \mathrm{d} F(\gamma)
$$

Note that flexibility is attractive when all restructurings are relatively valuable ( $\Gamma$ high), either because the fixed cost $K$ of adaptation to a new culture is low or because the productivity loss $\Delta^{\prime}$ under no restructuring is high. Conversely, gambling is desirable when the opportunity cost of replacing screened agents after a culturally incompatible restructuring is significant ( $G_{T}$ high). This cost may be high because the organization is old and agents have been screened for many periods ( $T$ high), because agents who have already been screened are very valuable ( $\Delta$ high) or because it is difficult to screen agents ( $p$ low).

\section{Cultural Structure and Incentives}

We have shown that inertia increases with the organization's age and uniformity (Propositions 1 and 2), and we have built a case for the optimality of cultural diversity (Proposition 3(b)). We now use it as a benchmark to present the next argument of the article, which is that an organization's cultural structure can mitigate or exacerbate internal incentive problems. We introduce the third main feature of our model, namely that to "fit in", agents have to make investments that are, to some extent, culture specific.

Culture-specific investment. Suppose now that all agents in the labor market are initially misfits, but can incur a nonobservable cost $c$ to become good fits for culture $A$ with probability $s$. Alternatively, they can incur $c$ to become good fits for culture $B$ with probability $s$. We can think of this cost as being that of having to form new habits, of acquiring certain social skills, etc. Note that 
the investment is not entirely culture specific: by investing in becoming a good fit for culture $A$ with probability $s$, the agent also becomes a good fit for culture $B$ with probability $s \mu$. However, the investment is partly culture specific: its return is lower if the agent operates in culture $B$ than if he operates in culture $A$ (i.e., $\mu<1$ ).

To clarify the discussion, we focus on the case in which our main points arise most naturally.

Assumption 1. $\Pi_{T}(1)<0$ and $\Pi_{T}\left(\frac{1}{2}\right)>\frac{1}{2} \Pi_{T}(0)$.

The first part of the assumption means that if all agents work under the same culture, then a culturally incompatible restructuring is not profitable. The second part of the assumption together with Proposition 3 implies that, if $c=0$, then it is optimal for the organization to maintain both cultures $\left(\alpha^{*}=1 / 2\right)$, and to restructure irrespective of whether $C=A^{\prime}$ or $C=B^{\prime} .^{17}$

Because the investment is not observable, it is not possible to sign contracts requiring explicitly the agents to undertake it. ${ }^{18}$ Therefore, one needs to study the agents' implicit incentives to make the investment. Of course, given Assumption 1 the problem does not arise when $c=0$, which corresponds to the case analyzed in previous sections. Assume now a strictly positive investment $\operatorname{cost} c$, and consider an agent set to work under culture $A$. Suppose first that the organization does not restructure if $C=B^{\prime}$. The agent's expected payoffs if he is a good fit for culture $A$ and if he is a misfit are, respectively,

$\frac{w}{1-\delta \lambda}$ and $\frac{w}{1-\delta \lambda(1-p)}$.

Hence, the expected increase in payoff from his investment in becoming a good fit is

$$
W_{1} \equiv\left(\frac{1}{1-\delta \lambda}-\frac{1}{1-\delta \lambda(1-p)}\right) w s .
$$

In what follows, we assume that agents find it optimal to incur the cost of becoming a good fit if this investment is not jeopardized by possible task restructurings.

Assumption 2. $0<c<W_{1}$.

Suppose now that the organization adapts to any environment change. The probability that an agent working under culture $A$ is "affected" by the

17. The constraints $\Pi_{T}(1)<0$ and $\Pi_{T}\left(\frac{1}{2}\right)>\frac{1}{2} \Pi_{T}(0)$ are compatible by concavity of $\Pi_{T}$. (Note that concavity of $\Pi_{T}$ is a result of the model not an assumption).

18. It would be enough that the investment be nonverifiable. Nonobservability of the investment also prevents selective firings and hirings. 
restructuring when $C=B^{\prime}$ (i.e., fired or reallocated to $B^{\prime}$ depending on whether the radical or gradual strategy is implemented) is

$z(\alpha) \equiv \frac{\int_{1-\alpha}^{1}(\gamma-(1-\alpha)) \mathrm{d} F(\gamma)}{\alpha}$

where the numerator represents the expected fraction of agents affected by the restructuring, and the denominator is the total fraction of agents working under culture $A$. If restructuring in period $T$ occurs whether $C=A^{\prime}$ or $C=B^{\prime}$ and the restructured tasks are filled with agents from the labor market (the radical strategy), the expected increase in the agent's wage from his investment in period 0 is

$\left(1-\frac{z(\alpha)}{2}\right) W_{1}+\frac{z(\alpha)}{2}\left(\frac{1-\delta^{T} \lambda^{T}}{1-\delta \lambda}-\frac{1-\delta^{T} \lambda^{T}(1-p)^{T}}{1-\delta \lambda(1-p)}\right) w s$.

The first term in the expression is the probability that the agent will not be affected by the restructuring, multiplied by $W_{1}$, the gain of investing under this condition. The second term is the likelihood of being affected, multiplied by the short-term gain of being skilled (i.e., wage times the increase in discounted expected length of employment until the new culture is adopted at $T$ and the agent is replaced).

If the organization adapts to the environment change with the gradual strategy, the expected increase in the agent's payoff from investing in becoming a good fit is

$$
\begin{aligned}
& \left(1-(1-\mu) \frac{z(\alpha)}{2}\right) W_{1} \\
& \quad+(1-\mu) \frac{z(\alpha)}{2}\left(\frac{1-\delta^{T} \lambda^{T}}{1-\delta \lambda}-\frac{1-\delta^{T} \lambda^{T}(1-p)^{T}}{1-\delta \lambda(1-p)}\right) w s
\end{aligned}
$$

The only difference compared to the radical strategy is that a good fit for culture $A$ who is reallocated to $B^{\prime}$ remains also a good fit for culture $B^{\prime}$ with probability $\mu$. Using equations (10)-(12) and rearranging terms, we can finally determine $W_{2}(\alpha, T)$, the increase in payoff from investing in becoming a good fit for culture $A$ when the organization adapts to the environment change if $C=B^{\prime}$

$W_{2}(\alpha, T)=W_{1}-\left(1-\mathbf{1}_{\mu>s} \mu\right) \frac{z(\alpha)}{2}\left(\frac{\delta^{T} \lambda^{T}}{1-\delta \lambda}-\frac{\delta^{T} \lambda^{T}(1-p)^{T}}{1-\delta \lambda(1-p)}\right) w s$,

where $\mathbf{1}_{\mu>s}=1$ if $\mu>s$ (the gradual strategy is adopted) and $\mathbf{1}_{\mu>s}=0$ if $\mu<s$ (the radical strategy is adopted). Note that $\partial W_{2}(\alpha, T) / \partial T>0$ and $W_{2}(\alpha, \infty)=W_{1}$, so that $W_{2}(\alpha, T)<W_{1}$ for all $T .{ }^{19}$ In words, the possibility

19. By symmetry, for agents working under culture $B$, the probability of being affected by a restructuring when $C=A^{\prime}$ is $z(1-\alpha)$ and the payoff increase from investing in becoming a good fit for $B$ is $W_{2}(1-\alpha, T)$. 
of a culturally incompatible task restructuring decreases the value for agents of investing in becoming a good fit. But, as we show below, the incentives of agents to undertake culture-specific investments depend, in turn, on the cultural structure of the organization.

Proposition 4 (Cultural structure and incentives). As $\alpha$ increases, the cost for an agent under $A$ of a culturally incompatible restructuring increases $\left(z^{\prime}(\alpha)>0\right)$, and so does his willingness to undertake an investment specific to culture $A$.

The analysis above has some important implications. Suppose for the time being that the cultural structure is fixed and exogenously given (Proposition 5 deals precisely with the endogeneity of cultural choice). In organizations in which multiple cultures coexist, agents have weaker incentives to undertake culture-specific investments. Conversely, cultural uniformity is more conducive to such investments. Therefore, the analysis predicts that incentive problems are more likely to manifest themselves in culturally diverse organizations. This also implies that it will be particularly important for culturally diverse organizations to spend effort and resources dealing with incentive problems. $^{20}$ They should provide incentives to their agents through incentive schemes (e.g., based explicitly on investment), intense screening of new and existing agents, and to reduce the investment cost for their agents, for example, through training programs or subsidies.

Interestingly, however, culturally diverse organizations may find themselves in a bind. Other things equal, the increased motivation of one group of agents decreases that of other groups. The investment of agents operating in culture $B$ reduces the organization's cost of adapting to a change when $C=B^{\prime}$, and therefore makes such a restructuring more likely. This, in turn, reduces the incentives of agents operating under culture $A$ (see also Section 5.1.2). The result also suggests that culturally diverse organizations will try to find ways to commit themselves to refrain from adapting to an environmental change. Such commitment may take the form of explicit contracts or more generally of other actions that increase the cost of adapting.

Naturally, the cultural structure is itself a choice variable. Proposition 4 has then implications for the optimal cultural mix within the organization. It implies that the gains associated with cultural diversity must be weighted against the costs of reduced incentives. In our setup, the costs and benefits of cultural uniformity are two sides of the same coin. On the one hand, cultural uniformity increases the expected cost of adapting to an environment change. Hence, if adapting is likely to be necessary, cultural diversity can be preferable. In other words, cultural diversity can be beneficial in that it can give the organization a greater ability to adapt to new contexts. On the other hand,

20. Rob and Zemsky (2002) study the relation between incentives and culture from a different angle. In their article, the organization sets an incentive mechanism that promotes the optimal level of cooperation and therefore the optimal accumulation of social capital. 
however, cultural uniformity can also be beneficial: the cultural inertia associated with cultural uniformity protects and thus encourages agents' culturespecific investments. As we have argued above, this is particularly important when the organization has no other (or no cheaper) way to commit not to adapt to an environmental change. Therefore, as incentive problems increase, so does the attractiveness of cultural uniformity for the organization. In fact, we show that cultural uniformity within organizations can arise as an optimal response to incentive problems.

Proposition 5 (Uniformity as commitment). There exists a threshold $c^{*} \in$ $\left(0, W_{1}\right)$ such that if $c \geq c^{*}$, a uniform culture is optimal $\left(\alpha^{*} \in\{0,1\}\right)$ and only a culturally compatible restructuring is undertaken (only when $C=A^{\prime}$ if $\alpha^{*}=1$ and only when $C=B^{\prime}$ if $\alpha^{*}=0$ ).

\section{Proof. See Appendix A4.}

Recall that, under Assumption 1, diversity is optimal in the absence of incentive problems (i.e., for $c=0$ ). Consider now the case of severe incentive problems. For example, if $c>W_{2}(\alpha, T)$, no agent will invest at any date to become a good fit for culture $A$ unless the organization commits not to restructure if $C=B^{\prime}$. Because explicit commitments are not feasible, the organization must set $\alpha$ large enough so that, when the time of the environment change arrives, it is not ex post profitable to restructure if $C=B^{\prime}$. Given that only culturally compatible restructuring are undertaken, the organization optimally sets maximum cultural uniformity, that is, all agents working under $A(\alpha=1)$. In other words, the inertia associated with sufficient cultural uniformity ex post locks the organization and thus encourages ex ante culture-specific investments by its agents.

Note that time also contributes to the commitment against opportunistic behavior. Recall that the organization's proportion of good fits increases over time (Proposition 1). Therefore, in case of uncertainty over $T$, cultural uniformity also serves as a commitment against culturally incompatible restructuring. In this case, the commitment will be imperfect; despite cultural uniformity, the organization may adapt to changes if they occur early enough in the organization's life. Nevertheless, cultural uniformity minimizes the probability of such an event.

\section{Applications}

Our theory argues that cultural uniformity can be an optimal response to incentive problems within an organization. More generally, uniformity will be conducive to culture-specific investments by their agents. The result has several implications, some of which are explored below. ${ }^{21}$

21. This section is more informal than the core of the article. It can be skipped by the reader interested only in a formal, analytical approach to the problem. 


\subsection{Internal Conflicts over the Management of Culture}

Culture is often at the center of conflicts internal to organizations. In our model, an active management of organizational culture is key to controlling the tensions between the organization's performance and the welfare and incentives of its agents. We illustrate the nature of potential conflicts with two examples: the choice of screening intensity and the hiring policy.

5.1.1 Organization versus Agents. The organization and its agents can disagree over the intensity of screening. Screening accelerates the accumulation of organization capital which, if managed properly, can never hurt the organization. Therefore, as long as it is free, screening is always beneficial for organizations. The agents' viewpoint is different. Intense screening forces them to incur the cost of becoming a good fit. Moreover, screening increases the likelihood of early firing of misfits, irrespective of whether they did not invest or their investment failed. Therefore, it may seem that the agents would favor an intensity of screening as low as possible. This presumption need not be true.

Result 3 (Conflict 1). The optimal intensity of screening from the agents' viewpoint is strictly positive if $c$ and $T$ are small enough. If screening involves a cost (partly) borne by the organization, then agents may prefer more screening than optimal from the organization's viewpoint.

\section{Proof. See Appendix A5.}

From the agents' viewpoint, screening has costs but also has benefits. Intense screening increases the likelihood of being fired for being a misfit. However, it also improves the organization's cultural fit and thus increases inertia. $^{22}$ When the cultural change is imminent ( $T$ small), agents are better-off accelerating the lock-in process. Conversely, when becoming a good fit is costly ( $c$ large), the benefits of a quite life under slack screening dominate. Hence agents may prefer more screening than the organization when the latter pays for it.

The organization's hiring policy can give rise to similar conflicts. Since uniformity protects their investment, agents may put pressure to set a hiring policy that results in greater cultural uniformity than optimal from the organization's viewpoint. Moreover, agents in place will also favor a more intense screening than optimal from the organization's viewpoint. Note that despite these conflicts, the organization may also have good reasons to delegate some control of the hiring policy if the agents' expertise is needed. For instance, recruiting can rely on agents identifying potential good hires so that, even

22. This is a variation on the "club effect". Ex ante, applicants to a top Ph.D. program wish it were not so selective. Ex post, however, those who have been admitted are not only happy that the hurdle was not lower but also want to increase it for future generations (see Result 5 below). 
if agents have no formal say in the hiring decision, they can recommend candidates. Also, the productivity of new recruits may depend on their colleagues' willingness to cooperate and share information (see Aghion and Tirole 1997).

5.1.2 Agents of Different Cultures. Conflicts can also arise between different groups of agents within the organization. Consider, for instance, a culturally diverse organization in which there is uncertainty about the environment change (i.e., about $\Delta^{\prime}, T$, or $K$ ). Let $p_{A}$ and $p_{B}$ denote the intensity with which agents working under cultures $A$ and $B$ are screened.

Result 4 (Conflict, 2). Relative to agents working under culture $B$, agents working under culture $A$ favor higher values of $p_{A}$ and lower values of $p_{B}$.

As the screening intensity of agents under culture $A$ increases, the opportunity cost of restructuring if $C=A^{\prime}$ decreases, which makes such a change more likely (the organization will adapt for higher $K$, lower $\Delta^{\prime}$, and lower $T$ ). This in turn makes agents working under culture $B$ worse-off. On the other hand, agents under culture $A$ are now better-off because the organization will set higher hurdles for restructuring if $C=B^{\prime}$. The result then suggests that multicultural organizations may be prone to tensions centered on the control of the hiring policy (see also Rotemberg and Saloner 1995; Rajan and Zingales 2000).

5.1.3 Agents of Different Age. Finally, conflicts are also likely to emerge between generations. Older generations of agents have been screened for longer so, conditional on having survived, their likelihood of being a misfit is lower than that of newer generations. Hence, independently of whether agents know if they are good fits or not, older agents are relatively less concerned with the possibility of a layoff for being misfits and therefore will favor more intense screening than their younger peers.

Result 5 (Conflict 3). Suppose that the level of screening is chosen by one generation of agents but applies to all current and future generations. Older agents will favor a more intense screening than younger ones.

\subsection{Expanding Organizations}

Concerns about an organization's culture are often raised at times of expansion, and in particular during mergers. In our framework, cultural identity can be endangered when the size of an organization evolves over time.

5.2.1 Growth. Consider an organization growing at rate $r$, that is, employing a mass $(1+r)^{t}$ of agents in period $t$ (for consistency, we impose the restriction that $\delta<\frac{1}{1+r}$ ). Assume that the organization grows by hiring agents from the labor market. Growth has two limiting effects on the organization's commitment ability not to undertake culturally incompatible restructuring. First, old and well-screened agents are "diluted" by the influx of new unscreened agents. Second, faster growing organizations put a greater weight on future payoffs. 
This increases the attractiveness of adopting new, more efficient cultures. Both the dilution effect and the long-term concern effect conflict with cultural inertia (see Proposition 1). However, we know that inertia can also be valuable. Therefore, if growth is a choice variable, the organization will trade-off the gains from faster growth against the commitment value of cultural inertia and uniformity.

Result 6 (Limits to growth). An organization may find it optimal to limit its growth rate as a commitment not to undertake culturally incompatible restructurings. Cultural uniformity will be more important for faster growing organizations.

\section{Proof. See Appendix A6.}

The organization might commit not to exploit all its growth opportunities by keeping away from certain lines of business, or developing a reputation for controlled growth. ${ }^{23}$ Another commitment tool is the organization's degree of cultural uniformity. To sustain fast growth, the organization may be led to trade-off cultural diversity against the commitment value of cultural uniformity. At a more general level, our theory suggests that periods of intense growth are critical to the organization's stability. If not carefully managed, growth can destroy cultural identity and exacerbate incentive problems within the organization. Finally, an aggravating factor may be the scarcity of suitable agents on the labor market. A growing organization might be forced to reduce its standards so as to hire enough new agents. This, in turn, reduces the agents' incentives to invest in fitting in the organization's culture.

5.2.2 Mergers. The difficulties encountered by some mergers, and even their failure, are sometimes attributed to "cultural incompatibilities". Consider two organizations $(i \in\{1,2\})$ identical in size, age, etc. merging in period 0. Suppose that both are culturally uniform, with $C_{i} \in\{A, B\}$ and that an environment change occurs at date $T$, with $C \in\left\{A^{\prime}, B^{\prime}\right\}$ as in our basic model. Does the merger generate more value when organizations are culturally similar $\left(C_{1}=\right.$ $C_{2}$ ) or when they are culturally different $\left(C_{1} \neq C_{2}\right)$ ? If the two organizations are culturally different, the merged organization is such that $\alpha=1 / 2$. Under Assumption 1, this cultural structure is optimal absent in incentive problems. Things are different, however, with more severe incentive problems.

Result 7 (Merger profitability). There exists a threshold $\hat{c}$ such that if $c \geq \hat{c}$ then a merger is more valuable when both organizations have similar rather than different cultures (i.e., $C_{1}=C_{2}$ rather than $C_{1} \neq C_{2}$ ).

23. Building a reputation for controlling growth may be easier that building one for not opportunistically undertaking culturally incompatible changes. Indeed, the opportunities to show restraint in growth strategy may be relatively frequent while instances in which a culturally incompatible change is an option are likely to be rare. 
From Proposition 5, we know that when incentive problems are severe, organizations are better-off with uniform cultures. The same logic naturally extends to the case of mergers. If two organizations with different cultures merge, the new organization is more likely to adapt to changed environmental conditions. This can be profitable but it may also reduce the incentives of its agents to undertake culture-specific investments.

\subsection{Other Applications}

In this section, we develop informally some possible extensions of our theory. ${ }^{24}$

5.3.1 Choosing the Investment Intensity. The agents' investment decisions involve externalities. For instance, consider a variation of our model in which the probability of becoming a good fit is monotonically increasing in the investment incurred. If the agents working under culture $A$ increase their investment level $s$, the opportunity cost of a restructuring when $C=B^{\prime}$ increases, making such restructuring less likely. Hence, the choice of a greater $s$ by his peers will make the investment of an agent working under culture $A$ more secure. This will encourage him also to invest more. This complementarity between the investment of agents working under the same culture has two implications. First, there is a multiplier effect of uniformity. Second, multiple equilibria can arise. $^{25}$

5.3.2 Specialists versus Generalists. The externality among agents concerns not only the level but also the nature of their investments. Suppose that agents can either invest in becoming "specialists" (i.e., they maximize the probability of being good fits for the current culture) or invest in becoming "generalists" (i.e., less effective in any given culture but more adaptable to other cultures). In terms of our model, there is a trade-off between $s$ and $\mu$. The organization's opportunity cost of undertaking a culturally incompatible restructuring is lower if its agents are generalists. Hence, an agent's incentive to invest in becoming a specialist increases if other agents are specialists, whereas his incentive to become a generalist increases if other agents are also generalists. In other words, differences in the nature of investments also give rise to multiple equilibria.

5.3.3 Reputation. One way for the organization to commit not to behave opportunistically is to develop a reputation for fairness. Kreps (1990) argues that

24. The extensions in Sections 5.3.1 and 5.3.2 are treated formally in Carrillo and Gromb (1999).

25. Acemoglu (1997) shows a similar multiple equilibria result when human capital and technology are complements: either workers train and firms adopt new technologies or they do not, and firms keep inefficient technologies. 
culture, defined as a set of messages stating how the organization will react to circumstances as they arise, may help in sustaining this reputation. In this context, reputation remains intact as long as there is an ex post agreement about which rule was meant to be applied in each situation, that is, culture facilitates the evaluation of which decisions are "fair" (see also Gibbons 1998). But Kreps' analysis does not explain how reputation is developed in the first place. One can argue that reputation is built through time, by the organization repeatedly refraining from acting opportunistically. According to our model, reputation building is accelerated if, at each period, more agents are in a position to be held up. As before, uniformity then helps in the commitment not to behave opportunistically.

5.3.4 Core Business. Many organizations are reluctant to undertake ventures outside their core business. Moreover, even if the organization is diversified, the dominant culture is usually related to its core business. There are several explanations for this phenomenon within the context of our framework. First, the initial culture might have been that of the core business, and by inertia the whole organization was developed around it. Second, agents working in the core business may be less substitutable. Therefore, insuring them against opportunistic behavior through cultural uniformity is more important. Last, agents in the core business may be more senior or more key to the organization. Their greater influence over the management of the organization's culture results in a hiring policy directed to the protection of their own culture-specific investments.

\section{Concluding Remarks}

It has been well established in modern Theory of Organizations that the behavior of individuals within an organization influences that of their peers even in the absence of direct externalities. This in turn shapes the organization's long-run performance, long after these particular agents have left it. Tirole (1996) proposes as an example of such "group phenomenon" the case of individuals whose behavior perpetuates the existing reputation (for quality, honesty, etc.) of their group vis-à-vis outsiders. This power of influence may harm the organization's performance, as in the case of herd behavior in a career concerns context (Scharfstein and Stein 1990) or improve it, as in the case of long-run cooperation between overlapping generations of agents (Crémer 1986; Kreps 1990). In this perspective, our contribution is to show that a more homogeneous cultural structure provides the organization's agents with better incentives to undertake culture-specific investments, which improves the organization's performance.

The article has some interesting empirical implications that one could test. Suppose that an industry has recently experienced an exogenous contextual change (deregulation, increased competition from abroad, etc.). Our theory predicts that younger organizations should be more malleable and therefore succeed better in the new environment than older ones (Proposition 1). By 
contrast, firms with a homogeneous culture are likely to suffer the most from the contextual change (Proposition 2). Note, however, the difficulty to evaluate empirically the degree of homogeneity of an organization. In this respect, it is probably easier to determine whether two merging organizations have homogeneous cultures or not. The prediction of our theory is that mergers of firms with similar cultures will have more problems to adapt to a change (Result 7). Last, and again under the caveat previously mentioned, industries with culturally homogeneous organizations should exhibit greater cultural disparity across organizations (Propositions 2 and 3).

Our analysis has abstracted from factors that might reinforce our results. For instance, cultural uniformity has a further commitment value if the organization can influence the likelihood of an environment change. Suppose that the organization can develop alternative personnel management methods, authority allocation, communication channels, etc. that may result in a new culture being developed. As the mass of agents working under one culture increases, the organization will devote more resources to developing alternatives which build on that culture and less to developing those that would constitute culturally incompatible changes. This results in such a change being less likely, and hence encourages investments in being a good fit for the existing culture. Furthermore, our model might underestimate the benefits of cultural uniformity by ignoring the possibility that if the organization does not protect its agents against its own opportunistic behavior, the agents may resort to other, more inefficient entrenchment methods.

Much work remains to be done. In particular, to focus our discussion, we have left aside issues such as why and how cultures emerge in the first place and what is embodied in the term culture. Also, our view of cultural structure as part of an incentive scheme begs the question of how it relates to other incentive tools such as pay-per-performance, for example. Last, one might want to relax some of our model's assumptions. As pointed by Schein (1986b), culture is reflected in many dimensions: choice of financial goals, control system, capital structure, etc. Hence, the restriction to two cultures may be too simplistic. This raises new interesting questions. For instance, it might be optimal for a diversified and well-established organization to focus on a subset of all potential cultures and, at the same time, adopt a balanced allocation of agents among these cultures. We leave these and other issues for future research.

\section{Appendix}

A1. Proof of Proposition 1

Given $\frac{\partial s_{T}}{\partial T}>0$, it is immediate that $\frac{\partial \Gamma-G_{T} E[\gamma]}{\partial T}<0$. As for the discount rate, we have

$$
\frac{\partial \Gamma-G_{T} E[\gamma]}{\partial \delta}=\frac{1}{[1-\delta]^{2}} \Delta^{\prime} E[\gamma]-\frac{1-\max \{s, \mu\}}{1-s} \frac{\left(s_{T}-s\right) \lambda(1-p s)}{[1-\delta \lambda(1-p s)]^{2}} \Delta E[\gamma]
$$

which is always positive given $\Delta^{\prime}>\Delta$. 
A2. Proof of Proposition 2

The net effect of an increase in the mass $\alpha$ of agents working under $A$ on the value of restructuring when $C=B^{\prime}$ is

$\frac{\partial \Pi_{T}(\alpha)}{\partial \alpha}=-G_{T}(1-F(1-\alpha))<0$.

The argument for the effect of an increase in the mass $1-\alpha$ of agents under $B$ on the value of restructuring when $C=A^{\prime}$ is identical.

\section{A3. Proof of Proposition 3}

Suppose first that the organization restructures irrespective of whether $C=A^{\prime}$ or $C=B^{\prime}$. The optimal cultural mix is then $\alpha^{*}=$ $\arg \max _{\alpha}\left\{\frac{1}{2} \Pi_{T}(\alpha)+\frac{1}{2} \Pi_{T}(1-\alpha)\right\}$. The first order condition gives

$\frac{1}{2} \Pi_{T}^{\prime}(\alpha)-\frac{1}{2} \Pi_{T}^{\prime}(1-\alpha)=0 \Leftrightarrow \frac{1}{2} G_{T}(F(1-\alpha)-F(\alpha))=0$,

which is solved for $\alpha^{*}=1 / 2$. Note that the second order condition is satisfied

$\frac{1}{2} \Pi_{T}^{\prime \prime}(\alpha)+\frac{1}{2} \Pi_{T}^{\prime \prime}(1-\alpha)=-\frac{1}{2} G_{T}\left(F^{\prime}(1-\alpha)+F^{\prime}(\alpha)\right)<0$.

Therefore, the maximal payoff conditional on restructuring if $C=A^{\prime}$ or $C=$ $B^{\prime}$ is $\Pi_{T}\left(\frac{1}{2}\right)$.

Second, given $\partial \Pi_{T}(\alpha) / \partial \alpha<0$, the optimal cultural mix conditional on restructuring only if $C=B^{\prime}$ is $\alpha^{*}=0$. Its corresponding expected payoff is $\frac{1}{2} \Pi_{T}(0)$, and the result follows.

\section{A4. Proof of Proposition 5}

First notice that for all parameter values, there is an equilibrium in which no agent invests. However, we will show that other equilibria always exist and Pareto dominates this one, which we will thus rule out. The organization can always secure $\Pi_{T}(0)$ by setting $\alpha \in\{0,1\}$. The question is whether it can do better under cultural diversity. If $c \geq c^{*} \equiv W_{2}(1 / 2, T)$, then agents in periods $\tau=0,1, \ldots, T-1$ do not invest unless they anticipate no culturally incompatible restructuring. Therefore, conditional on undertaking only culturally compatible restructurings, it is optimal for the organization to set all agents under the same culture, $\alpha^{*} \in\{0,1\}$.

For completeness, we also analyze the case $c<c^{*}$.

- If $c$ is small enough, the incentive constraint is not binding. That is, all agents are willing to invest to fit into the culture despite the risk of their investment becoming obsolete. In this case, and given Assumption 1, flexibility is the organization's optimal strategy. Formally, if $c \leq W_{2}(1 / 2,1)$, then agents hired in period $\tau=T-1$ invest despite the risk of becoming redundant at $T$. Since $\partial W_{2}(1 / 2, t) / \partial t>0$, agents in periods 
$T-2, T-3, \ldots, 0$ also invest. In this case, the incentive constraint is slack and so, from Proposition 3 and Assumption 1, $\alpha^{*}=1 / 2$.

- If $W_{2}(1 / 2,1)<c<c^{*}$, then the incentive constraint is slack only for some agents (those who enter the organization in periods far enough from $T$ ). Therefore, only these individuals are willing to invest despite the possibility of a cultural change. Which strategy - flexibility or gamblingdominates, in this case, will crucially depend on how large that fraction of agents is. Formally, for all $\alpha$ there exist $\tau_{F}^{*} \in(0, T)$ such that only agents hired before $T-\tau_{F}^{*}$ invest in becoming good fits for culture $F \in\{A, B\}$. In this case, the organization faces two possibilities. First, to restructure when $C=A^{\prime}$ and $C=B^{\prime}$ (flexibility). Then, $\alpha^{*} \in\{0,1\}$ (otherwise, either $\Pi_{T}\left(\alpha^{*}\right)<0$ or $\Pi_{T}\left(1-\alpha^{*}\right)<0$ ). However, $\alpha^{*}$ need not be $1 / 2$. Second, to make only culturally compatible restructuring (gambling), and therefore set $\alpha^{*} \in\{0,1\}$. Note that the organization's payoff under flexibility decreases as $c$ increases (technically, for any given $\alpha, \tau_{A}^{*}$ and $\tau_{B}^{*}$ are step functions decreasing in $c$, which simply means that agents are relatively less willing to invest the higher the cost). Overall, whether flexibility or gambling is optimal will depend on $c$. In particular, for $c \rightarrow c^{*}$ then $\alpha^{*} \in\{0,1\}$ and for $c \rightarrow W_{2}(1 / 2,1)$ then $\alpha^{*}=1 / 2$.

\section{A5. Proof of Result 3}

Consider an organization with all agents working under culture $A$. For a given $p$, denote by $Y_{N}(p)$ the payoff of an agent when he does not invest and the organization adapts if $C=A^{\prime}$ or $C=B^{\prime}$, and by $Y_{I}(p)-c$ his payoff if he invests and the organization adapts only if $C=A^{\prime}$

$$
\begin{aligned}
Y_{N}(p) & =w\left[\frac{1}{1-\delta \lambda(1-p)}-\frac{z(\alpha)}{2} \times \frac{\delta^{T} \lambda^{T}(1-p)^{T}}{1-\delta \lambda(1-p)}\right], \\
Y_{I}(p)-c & =w\left[\frac{s}{1-\delta \lambda}+\frac{1-s}{1-\delta \lambda(1-p)}\right]-c .
\end{aligned}
$$

Other things equal, the agent prefers less screening $\left(\partial Y_{I} / \partial p<0\right.$ and $\left.\partial Y_{N} / \partial p<0\right)$. However, he also benefits from the organization's commitment not to adapt if $C=B^{\prime}\left(Y_{I}(p)>Y_{N}(p)\right)$.

Now, for any given $c$, denote by $p^{*}$ the probability of detection such that $W_{1}\left(p^{*}\right)=c$, where $W_{1}(p)$ is defined by equation (10). ${ }^{26}$ Moreover, suppose that Assumption 1 holds for $p=p^{*}$.

1. If $p \in\left[0, p^{*}\right)$, then $c>W_{1}(p)$ : the agent does not invest independently of the organization's behavior. The organization's dominant strategy is then to adapt if $C=A^{\prime}$ or $C=B^{\prime}$. The agents' payoff is $Y_{N}(p)$, which is maximized at $p=0$. 
2. If $p \in\left[p^{*}, 1\right]$, then $c \leq W_{1}(p)$ : the agent invests if the organization undertakes only culturally compatible restructurings. Given Assumption 1, the organization's dominant strategy is then to adapt only if $C=A^{\prime}$. The agents' payoff is $Y_{I}(p)$, which is maximized at $p=p^{*}$.

Overall, agents optimally choose $p=p^{*}$ if $Y_{I}\left(p^{*}\right)>Y_{N}(0)$ and $p=0$ if $Y_{I}\left(p^{*}\right)<Y_{N}(0) .^{27}$

\section{A6. Proof of Result 6}

Suppose that $C=B^{\prime}$ and conduct the following thought experiment. Suppose that the organization stops growing after period $T$. In period $t \in\{1, \ldots, T\}$, the mass of agents is $(1+r)^{t}$ and the fraction of good fits is

$$
\hat{s}_{t}=\frac{1}{(1+r)^{t}}\left(s_{t}+r \sum_{\tau=0}^{t-1}(1+r)^{t-1-\tau} s_{\tau}\right) \text {. }
$$

For all $t$, the cultural fit $\hat{s}_{t}$ decreases with $r$ (i.e., $\left.\partial \hat{s}_{t} / \partial r<0\right): \hat{s}_{t}$ is a weighted average of $s_{\tau}$ with $\tau \in\{0, \ldots, t\}$, and it is easily checked that the relative weight on larger values of $\tau$ decreases as $r$ increases. Hence, the expected gain from adapting is

$$
(1+r)^{T}\left[\frac{\Delta^{\prime}}{1-\delta} E[\gamma]-\hat{G}_{T} \int_{1-\alpha}^{1}(\gamma-(1-\alpha)) \mathrm{d} F(\gamma)\right],
$$

where $\hat{G}_{T}$ is defined as $G_{T}$ in equation (7) replacing $s_{T}$ with $\hat{s}_{T}$. Consider now the effect of growth after period $T$. In period $T+\tau$, a mass $r(1+r)^{T+\tau-1}$ of new agents are hired, an expected fraction $E[\gamma]$ of which generates $\Delta^{\prime}$. Hence, considering these new agents only, the expected gain from adapting is

$$
\sum_{\tau=1}^{+\infty} \delta^{\tau} r(1+r)^{T+\tau-1} \Delta^{\prime} E[\gamma]
$$

Summing these terms, the organization's net expected gain from adapting is

$$
\begin{aligned}
\hat{\Pi}(\alpha, r, T)= & (1+r)^{T}\left[\frac{\Delta^{\prime}}{1-\delta} E[\gamma]-\hat{G}_{T} \int_{1-\alpha}^{1}(\gamma-(1-\alpha)) \mathrm{d} F(\gamma)\right] \\
& +\sum_{\tau=1}^{+\infty} \delta^{\tau} r(1+r)^{T+\tau-1} \Delta^{\prime} E[\gamma]-K
\end{aligned}
$$

That is, the organization adapts when $C=B^{\prime}$ if and only if $\hat{\Pi}>0$. Given that $\partial \hat{s}_{T} / \partial r<0$, it is easily shown that $\partial \hat{\Pi} / \partial r>0$. By Assumption 1 , we have that $\hat{\Pi}(\alpha=1, r=0, T)<0$. Besides, if $r \rightarrow(1-\delta) / \delta($ i.e., if $1 /(1+r) \rightarrow \delta)$,

27. In this model, the agents' optimal screening intensity is either 0 or $p^{*}$. This can be easily generalized. For instance, if the date of the environment change is uncertain, agents will choose (depending on $\Pi_{T}$ ) among several locally optimal positive levels of screening. 
then $\hat{\Pi} \rightarrow+\infty$. Hence, by continuity, there exists a unique $r_{\max }(\alpha, T)$ such that $\hat{\Pi}\left(\alpha, r_{\max }(\alpha, T), T\right)=0$. Note that since $\partial \hat{\Pi} / \partial \alpha<0$ we have $\partial r_{\max } / \partial a>0$ and $r_{\max }$ is maximal for $\alpha=1$.

\section{References}

Acemoglu, D. 1997. "Training and Innovation in an Imperfect Labor Market," 64 Review of Economic Studies 445-64.

Aghion, P., and J. Tirole. 1997. "Real and Formal Authority in Organizations," 105 Journal of Political Economy 1-29.

Barney, J.B. 1986. "Organizational Culture: Can It Be a Source of Sustained Competitive Advantage?" 11 Academy of Management Review 656-65.

Besanko, D., D. Dranove, and M. Shankley. 2000. The Economics of Strategy. 2nd ed. New York, N.Y.: Wiley.

Bourdieu, P. 1983. "Forms of Capital," in J.C. Richards, ed., Handbook of Theory and Research for the Sociology of Education. New York, N.Y.: Greenwood.

Boyer, M., and J. Robert. 2006. "Organizational Inertia and Dynamic Incentives," 59 Journal of Economic Behavior and Organization 324 48.

Brezis, E.S., P. Krugman, and D. Tsiddon. 1993. "Leapfrogging in International Competition: A Theory of Cycles in National Technological Leadership," 83 American Economic Review $1211-9$.

Carrillo, J.D. 2003. "Job Assignments as a Screening Device," 21 International Journal of Industrial Organization 881-905.

Carrillo, J.D., and D. Gromb. 1999. "On the Strength of Corporate Cultures," 43 European Economic Review, Papers and Proceedings 1021-37.

Cohen, D., and L. Prusak. 2001. In Good Company. How Social Capital Makes Organizations Work. Boston, Mass.: Harvard Business School Press.

Coleman, J.S. 1988. "Social Capital in the Creation of Human Capital," 94 American Journal of Sociology S95-120.

Crémer, J. 1986. "Cooperation in Ongoing Organizations," 51 Quarterly Journal of Economics $33-50$. 351-86.

1993. "Corporate Culture and Shared Knowledge," 2 Industrial and Corporate Change . 1995. Comment on Edward P. Lazear 'Corporate Culture and the Diffusion of Values', in H. Siebert, ed., Trends in Business Organization: Do Participation and Cooperation Increase Competitiveness? Tübingen: J.C.B; Mohr (Paul Siebeck).

Gibbons, R. 1998. "Game Theory and Garbage Cans: An Introduction to the Economics of Internal Organization," in J. Halpern and R. Stern, eds., Debating Rationality: Nonrational Elements of Organizational Decision Making, chap. 2. Ithaca, N.Y.: ILR Press.

Hirshleifer, D., and I. Welch. 2002. "An Economic Approach to the Psychology of Change: Amnesia, Inertia, and Impulsiveness," 11 Journal of Economics \& Management Strategy 379-421.

Kotter, J., and J. Heskett. 1992. Corporate Culture and Performance. New York, N.Y.: Free Press.

Kreps, D. 1990. "Corporate Culture and Economic Theory," in J. Alt and K. Shepsle, eds., Perspectives on Positive Political Economy. Cambridge: Cambridge University Press, 90-143.

Lazear, E. 1999. "Culture and Language," 107 Journal of Political Economy 95-126.

Prescott, E., and M. Visscher. 1980. "Organization Capital," 88 Journal of Political Economy 446-61.

Putnam, R.D. 2000. Bowling Alone. The Collapse and Revival of American Community. New York, N.Y.: Simon and Schuster.

Rajan, R., and L. Zingales. 2000. "The Tyranny of Inequality: An Enquiry Into the Adverse Consequences of Power Struggles," 76 Journal of Public Economics 512-58.

Rob, R., and P. Zemsky. 2002. "Social Capital, Corporate Culture, and Incentive Intensity," 33 Rand Journal of Economics 243-57. 
Rotemberg, J., and G. Saloner. 1994. "Benefits of Narrow Business Strategies," 84 American Economic Review 1330-49.

. 1995. "Overt Interfunctional Conflict (And Its Reduction Through Business Strategy)," 26 Rand Journal of Economics 630-53.

Scharfstein, D., and J. Stein. 1990. "Herd Behavior and Investment," 80 American Economic Review 465-79.

Schein, E. 1986a. "Are You Corporate Cultured?" 65 Personnel Journal 83-96.

1986b. "What You Need to Know About Organizational Culture," 40 Training and Development Journal 30-3.

Tirole, J. 1996. "A Theory of Collective Reputations (With Applications to the Persistence of Corruption and to Firm Quality)," 63 Review of Economic Studies 1-22.

Wilson, J.Q. 1989. Bureaucracy. What Government Agencies Do and Why They Do It. New York, N.Y.: Basic Books. 